\title{
Is V-Y plasty necessary for penile lengthening? Girth enhancement and increased length solely through circumcision: description of a novel technique
}

\author{
Nikos Mertziotis, Diomidis Kozyrakis and Elias Bogris
}

Our objective is to describe a novel ligamentolysis approach using a subcoronal incision technique and to determine its safety and efficacy. During the last 7 years, 82 consecutive patients had penile augmentation surgery. Ligamentolysis, through a lower abdominal incision (V-Y plasty) in the first 35 males, was performed (Group A), followed by circumcision ligamentolysis in the next 47 males (Group B). The operation time, complications, and the preoperative and postoperative values of penile length and girth along with the self-esteem and relations questionnaire score as well as satisfaction score was calculated before and after the surgery, and a comparison was conducted between the groups. The mean age at presentation was 32 years (range: 18-56 years). Seventy-nine patients suffered from penile dysmorphophobia, and three patients had micropenises (length $<7.5 \mathrm{~cm}$ ). The mean surgical times were 150.7 and 125.2 min for Groups A and B, respectively $(P=0.005)$. Postoperatively, four Group A patients and three Group B patients ( $11 \%$ versus $6 \%$, respectively) experienced penile retraction $(P=0.453)$. Hypertrophic scars were observed in 18 men $(51 \%)$ in the former group. In the circumcision group, no major wound complications were recorded. The length and girth improvements between the groups were similar. In terms of satisfaction and SEAR improvement, the resulting difference for both variables favored the circumcision group $(P=0.007$ and $<0.001$, respectively). With strict selection criteria, the circumcision ligamentolysis procedure compared to the $\mathrm{V}-\mathrm{Y}$ plasty demonstrated improved results in terms of safety, operation time, retraction rate and cosmetic appearance without any compromise in the gained penile size.

Asian Journal of Andrology (2013) 15, 819-823; doi:10.1038/aja.2013.58; published online 24 June 2013

Keywords: augmentation phalloplasty; suspensory ligament incision; V-Y plasty

\section{INTRODUCTION}

For centuries, penile size has been a source of major concern and anxiety to the male population. Despite that in the majority of men, penile length is within the normal range, the concern regarding its size may lead to low self-esteem, sexual dysfunction and even psychiatric disorders. The psychiatric term 'penile dysmorphophobia' has been introduced to describe the abnormal perception of penile size, although it has normal dimensions.

The majority of patients who request penile augmentation are dysmorphophobic rather than micropenile men. ${ }^{1,2}$ However, augmentation phalloplasty is not always successful and occasionally leads to penile shortening. ${ }^{3,4}$ Moreover, the reported satisfaction rates are often poor, which is most likely because the formation of even small hypertrophic scars or keloids for these patients can reduce their inclination for surgery. Therefore, detailed counseling regarding the postoperative results and surgical complications is of utmost importance. ${ }^{5}$ Several penile augmentation surgical techniques have been proposed, and the most widely performed is the incision of the suspensory ligament (ligamentolysis) combined with V-Y plasty. ${ }^{3}$ Herein, we introduce a new approach for penile ligamentolysis and our experience with the procedure when performed in 82 patients.

\section{MATERIALS AND METHODS}

Patient selection

All males requesting penile augmentation underwent a detailed medical evaluation and strict criteria were applied before proceeding to surgery. The investigation of the patients' medical history focused on urogenital, neurological, hormonal and psychosexual disorders. Patients with erectile dysfunction, urological diseases, central nervous system abnormalities and major psychological disorders were excluded as surgical candidates.

Subsequently, all males underwent a thorough physical examination, and patients with signs of hypogonadism were further investigated. The penile length was calculated from the base of the penis to the tip of the glans while in a stretched condition according to the technique proposed by Levine and Larsen. ${ }^{6}$ The girth was measured from the base of the penis while in a flaccid state. The above measurements were calculated before surgery and 12 months after surgery. A micropenis is defined as any penis with a size that is less than two standard deviations from the mean value, and based on epidemiologic reports, would be any penis that is less than $7.5 \mathrm{~cm}$ in length while stretched/erect. ${ }^{7}$

In contrast, dysmorphophobic patients have normal-sized penises, which will continue to be normal after any type of augmentation 
surgery. Importantly, we emphasized that all surgical candidates, particularly those with dysmorphophobic conditions, should be extensively informed about the definition of normal dimensions. Patients who insisted on surgical treatment were routinely examined by a psychiatrist. The presence of a major psychological disorder represented an exclusion criterion.

We attempted to evaluate the patients' preoperative self-esteem status, expectations and motivations. The validated erectile dysfunction 'self-esteem and relationship' questionnaire (SEAR) (Appendix 1) translated into Greek was very helpful. ${ }^{8}$ The quantification of patients' satisfaction was derived from the sum of questions 3, 7 and 14 of the SEAR questionnaire. Each patient answered the questionnaire before the operation and 12 months after the operation. Moreover, we developed and used in parallel a comprehensive questionnaire to identify and exclude ineligible surgical candidates (Appendix 2). The first three questions sought to elucidate the effect of the individual's small penis perception on personal relationships, self-esteem status and the expected improvement in the quality of life after the augmentation procedure. The possible answers are qualitative ones, such as yes, no and I cannot say.

The following two questions were used to quantify the candidate's expectations regarding the length and girth after augmentation. Those patients with unrealistic expectations and motivations, high self-esteem status and high rates of satisfaction, as defined by the SEAR questionnaire, were discouraged from surgical management. All patients were informed about the risks and the experimental and irreversible nature of the surgery, as well as the unpredictability of the procedure outcome. Informed consent was provided by all participants.

\section{Surgical technique}

In the first 35 patients, the ligamentolysis was performed through a lower abdomen incision. For skin advancement over the elongated penis, an inverted $\mathrm{V}-\mathrm{Y}$ plasty was initially the procedure of choice (Group A). Because of cosmetic imperfections (e.g., scars and keloids) associated with the abdominal incision, ligamentolysis in the next 47 patients was performed through a coronal sulcus incision (circumcision) followed by degloving of the penis up to its base to gain access to the suspensory ligament (Group B). To our knowledge, access to the suspensory ligament through circumcision for the purpose of penile elongation has never been described previously (Figure 1b-d).

To overcome the postsurgical reattachment of the penile shaft to the pubis, penile stretching was exercised as soon as possible, often in the second week post-surgery. Mechanical stretching with the use of a penis extender was then introduced by the sixth postoperative week, and this device was recommended to be used for several months. Because of the skin incision, the use of this device proved to be difficult for the Group A males, where the patient's compliance improved only after the healing of the wound. Using the penis extender was not problematic for the Group B patients, where the absence of the V-Y incision allowed the patients to start using the device earlier.

For girth augmentation purposes, a free dermal-fat flap harvested from the medial posterior area of the buttock-thigh creases was interposed circumferentially underneath the dartos fascia (excluding the ventral surface-corpus spongiosum), from the base of the penis to the coronal sulcus with the dermal surface lying just above the tunica albuginea. The flap was fixed to the tunica using 4.0 monofilament absorbable sutures (Figure 1e). The methods used to evaluate the surgical results were the improvement in penile size, the degree of patient's satisfaction and the improvement in the overall SEAR questionnaire score.

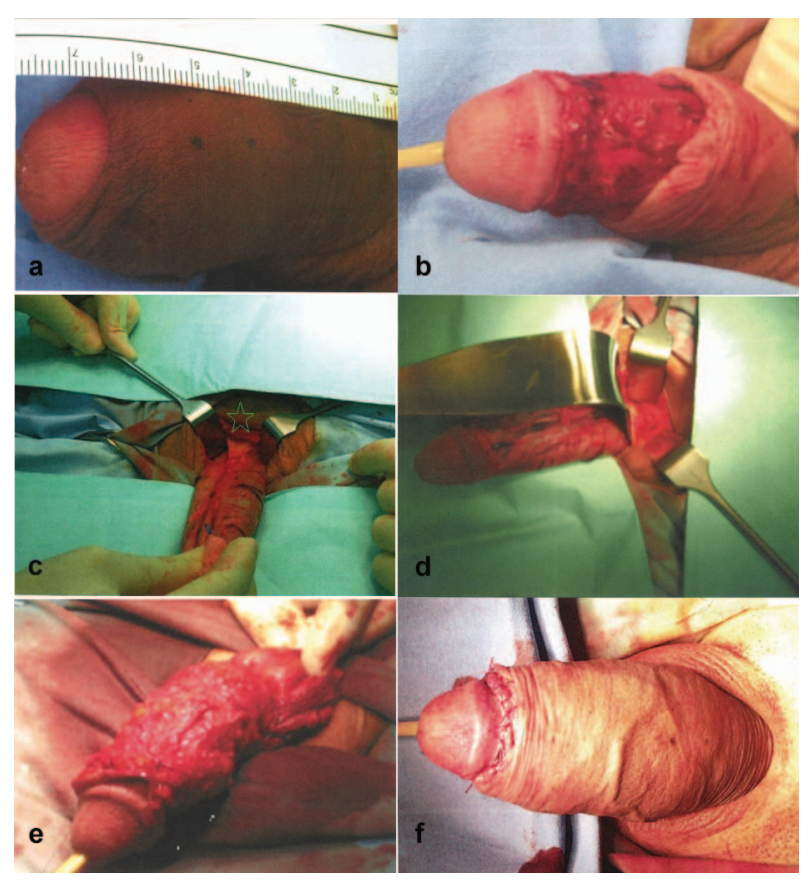

Figure 1 (a) Preoperative image; (b) subcoronal incision and degloving of the penile shaft; (c) suspensory ligament detection (asterisk); (d) suspensory ligament incision; (e) autologous free dermal-fat graft was fixed over the tunica albuginea; (f) final postoperative result.

\section{Statistical analysis}

The clinical characteristics of each group together with a comparison between them regarding the preoperative characteristics are presented in Table 1. The preoperative and postoperative values of penile length and girth and the satisfaction and SEAR scores were statistically examined using the Shapiro-Wilk test for normality (Table 2). To evaluate the outcome of our surgical techniques, a direct comparison of each postoperative parameter versus the respective baseline (preoperative) value was initially conducted separately for each group. This was followed by a comparative study of the mean changes between the two groups using the $\mathrm{V}-\mathrm{Y}$ plasty patients as control for the circumcised patients. Abnormally distributed variables were statistically examined using a non-parametric test (Mann-Whitney and Wilcoxon matched pairs signed-rank test), while parametric tests ( $t$-test and pairwise $t$ test) were applied to the rest of the variables (Table 3 ). The $t$-test was used for statistical analysis of the operative times. Fisher's exact test was

Table 1 Clinical characteristics and outcome for each group of patients

\begin{tabular}{lcc}
\hline Parameter & Group A & Group B \\
\hline Number of patients & 35 & 47 \\
Age (year), range & $27(18-53)$ & $35(20-56)$ \\
Operation time (min) & $150.7 \pm 22.25$ & $125.2 \pm 33.22$ \\
Preoperative length (cm) & $12.03 \pm 2.19$ & $12.31 \pm 2.85$ \\
Postoperative length (cm) & $13.95 \pm 3.17$ & $14.41 \pm 3.43$ \\
Preoperative girth (cm & $6.32 \pm 1.01$ & $6.25 \pm 1.20$ \\
Postoperative girth (cm) & $8.52 \pm 1.35$ & $8.24 \pm 1.26$ \\
Preoperative satisfaction & $7.66 \pm 2.14$ & $8.13 \pm 2.55$ \\
Postoperative satisfaction & $10.20 \pm 2.21$ & $12.15 \pm 2.19$ \\
Preoperative SEAR & $31.49 \pm 11.31$ & $28.55 \pm 6.93$ \\
Postoperative SEAR & $37.86 \pm 13.92$ & $43.94 \pm 11.07$ \\
\hline
\end{tabular}

Abbreviation: SEAR, Self-Esteem and Relationship. 
Table 2 Shapiro-Wilk test for normality. Variables with $P<0.05$ have abnormal distribution

\begin{tabular}{lc}
\hline & P value \\
\hline Group A & \\
Operation time & 0.55 \\
Length increase & $<0.001$ \\
Girth increase & 0.667 \\
Satisfaction improvement & 0.060 \\
SEAR improvement & 0.329 \\
Group B & \\
Operation time & 0.60 \\
Length increase & $<0.001$ \\
Girth increase & 0.303 \\
Satisfaction improvement & $>0.999$ \\
SEAR improvement & 0.823 \\
\hline
\end{tabular}

Abbreviation: SEAR, Self-Esteem and Relationship.

used to compare any wound complications and penile retractions (Table 4). Statistical significance was set at $P<0.05$. The statistical analysis was conducted using the PASW Statistics 18 (formerly SPSS statistics) software (SPSS Inc., Chicago, IL, USA).

\section{RESULTS}

During the last 7 years, 82 patients underwent suspensory ligament incision (ligamentolysis) for penile elongation in our department, and a concomitant girth augmentation was performed in 79 of these individuals.

The preoperative characteristics were similar between the two groups (Table 1). The mean age at presentation was 32 years (range: 18-56 years), whereas the mean preoperative stretched penile length for Groups A and B was $12.03 \mathrm{~cm}$ (range: $7.6-14.7 \mathrm{~cm}$ ) and $12.31 \mathrm{~cm}$ (range: $6.8-17.1 \mathrm{~cm}$ ), respectively (Table 1). Seventy-nine patients suffered from penile dysmorphophobia, while the remaining three had a true micropenis (less than $7.5 \mathrm{~cm}$ in length).

Table 3 Comparison of the preoperative (baseline) versus the postoperative value for each group separately and of the change of each value between the two groups

\begin{tabular}{lcc}
\hline Variable & Mean change (SD) & P value \\
\hline Group A & $1.92(1.48)$ & $<0.001^{\mathrm{a}}$ \\
Post length vs. Pre length & $2.21(1.11)$ & $<0.001^{\mathrm{b}}$ \\
Post girth vs. Pre girth & $2.54(0.82)$ & $<0.001^{\mathrm{b}}$ \\
Post satisfaction vs. Pre satisfaction & $6.37(0.73)$ & $<0.001^{\mathrm{b}}$ \\
Post SEAR vs. Pre SEAR & & \\
Group B & $2.11(2.71)$ & $<0.001^{\mathrm{a}}$ \\
Post length vs. Pre length & $2.00(2.22)$ & $<0.001^{\mathrm{b}}$ \\
Post girth vs. Pre girth & $4.02(4.43)$ & $<0.001^{\mathrm{b}}$ \\
Post satisfaction vs. Pre satisfaction & $15.38(9.10)$ & $<0.001^{\mathrm{b}}$ \\
Post SEAR vs. Pre SEAR & & \\
Comparison of changes between Groups A & & $0.815^{\mathrm{c}}$ \\
and B & -0.19 & $0.220^{\mathrm{d}}$ \\
Length increase & 0.21 & $0.007^{\mathrm{d}}$ \\
Girth increase & -1.48 & $<0.001^{\mathrm{c}}$ \\
Satisfaction improvement & -9.01 & \\
SEAR improvement & & \\
\hline
\end{tabular}

Abbreviation: SEAR, Self-Esteem and Relationship.

a Wilcoxon matched pairs signed-rank test.

${ }^{b}$ Pairwise $t$-test.

${ }^{c}$ Mann-Whitney $U$ test.

d t-test.
Table 4 Statistical analysis of operation times and complications between the two groups

\begin{tabular}{lccr}
\hline Value & Group A & Group B & P value \\
\hline Operation time (min) & 150.7 & 125.2 & $0.005^{\mathrm{a}}$ \\
Retraction (no. of patients) & $4(11 \%)$ & $3(6 \%)$ & $0.453^{\mathrm{b}}$ \\
$\begin{array}{l}\text { Wound complications (no. of } \\
\quad \text { patients) }\end{array}$ & $18(51 \%)$ & $0(0 \%)$ & $<0.001^{\mathrm{b}}$ \\
\end{tabular}

a $t$-test.

${ }^{\mathrm{b}}$ Fisher's exact test.

In the V-Y plasty group, the mean improvement of the penile length and girth was $1.92 \pm 1.48$ and $2.21 \pm 1.11 \mathrm{~cm}$ and was $2.11 \pm 2.71$ and $2.00 \pm 2.22 \mathrm{~cm}$, respectively, in the circumcision group. For each group, the improvement over the baseline was statistically significant $(P<0.001$ for all values). However, no significant improvement was revealed when comparing the two groups $(P=0.815$ and $P=0.220$ for length and girth increases, respectively) (Table 3 ).

The mean surgical time was 150 and $125 \mathrm{~min}$ for the $\mathrm{V}-\mathrm{Y}$ and circumcision techniques, respectively $(P=0.005)$. Postoperatively, four patients from Group A and 3 patients from Group B ( $11 \%$ versus $6 \%$, respectively) experienced penile retraction $(P=0.453)$. In the circumcision group, no major wound complications were recorded, whereas in the V-Y group, five obese patients experienced wound dehiscence and one was reoperated upon for debris removal. Overall, hypertrophic scars were observed in 18 patients (51\%) from this group (Table 4).

For Groups A and B, the improvement in the satisfaction score was 2.54 and 4.02 (both $P<0.001$ ) and the improvement of the SEAR was 6.37 and 15.38 , respectively $(P<0.001)$. Comparison of the two groups, in terms of satisfaction and SEAR improvement, revealed that the differences between each variable favored the circumcision group ( $P=0.007$ and $P<0.001$ respectively).

\section{DISCUSSION}

An indication that a patient may require penile augmentation is the presentation of a true micropenis, which is defined as a stretched penis length under $7.5 \mathrm{~cm} .{ }^{6}$ For those patients, surgical treatment could be considered after detailed counseling. In cases of dysmorphophobic patients who request penile augmentation, any type of surgery carries the risk of failure with potentially suboptimal results and unsatisfied patients. ${ }^{3-5}$ Given that these males often have an unrealistic perception of their penile size, their expectations from the surgical outcome are also often unrealistic. These individuals should be discouraged from any surgical intervention and should be evaluated psychologically before any operation. ${ }^{2,3}$ In our opinion, surgical treatment is the last resort and should be implemented only when all other conservative measures have failed.

The postsurgical benefit in length and girth in our patients was greater than, or at least similar to, other reports, after ligamentolysis combined with $\mathrm{V}-\mathrm{Y}$ plasty. In a review article, the mean penis length gain was $1-2 \mathrm{~cm}$ (range $0.75-4 \mathrm{~cm}$ ), and the girth gain was between 2.0 and $2.7 \mathrm{~cm} .{ }^{5}$ The major limitations in performing phalloplasty augmentations are the postsurgical complications and particularly the hypertrophic and wide scars, the hair-bearing skin flap, low hanging penis, infections, nodal formations and penile deformities. ${ }^{9-11}$ In our $\mathrm{V}-\mathrm{Y}$ plasty group, with the exception of five patients with wound breakdown, a high percentage of males $(51 \%)$ complained of postsurgical hypertrophic scars. In the circumcision group, this complication did not occur, and this was statistically reflected through the superiority of change of satisfaction and SEAR score for this group 
compared to the $\mathrm{V}-\mathrm{Y}$ patients, despite the fact that both techniques were equivalent in length and girth gain (Table 4).

As reported by some authors, another point of skepticism regarding ligamentolysis is the shortening of a preoperatively normal penis. ${ }^{3-5}$ This is caused primarily by the postoperative reattachment of the penile shaft to the pubis. To overcome this condition, the dermalfat pad was interposed in the pubic-penile space and penile weights and vacuum devices were applied. ${ }^{4}$ Others proposed the insertion of a silicon buffer. ${ }^{12}$ In our opinion, the penis extender device for penile traction is a preferable option because of its relatively low cost (approximately $€ 100$ ), its adjustability to any penile size, its ease in wear and the fact that high motivation is not a prerequisite for its success.

Overall, seven patients experienced penile retractions due to penile reattachment, but there was no significant difference between our two groups (Table 4). The rate of poorly satisfied patients has been reported to be as high as $62 \% .{ }^{5}$ Similarly, Li et al. ${ }^{12}$ noted that despite an adequate penile length gain, the satisfaction rates were low $(35 \%$ overall and $27 \%$ in the dysmorphophobic group). Other investigators published more favorable outcomes with $88 \%-91 \%$ of patients experiencing an improvement of self-esteem and functional status. ${ }^{13,14}$ Although a direct comparison of our study with other reports cannot be made, our patients demonstrated an improvement of satisfaction and self-esteem score over the preoperative baseline that was statistically significant in both groups, and this effect was more pronounced in Group B. To some extent, our strict inclusion criteria and our fivestep questionnaire together with the detailed preoperative information may have contributed to these results. However, we believe that these favorable outcomes are due primarily to the absence of surgical scars and less to the size achieved by the augmentation procedure. One could assume that these males who have a 'more normal appearance' feel more confident when they get undressed in front of their partner, and this may be reflected in the status of their self-esteem.

A criticism in using questionnaires is that they have been validated for the treatment of erectile dysfunction and not for augmentation procedures, which may have biased our results. The retrograde nature of the study and the difference in surgical expertise, as experience is accumulated over time, can also be considered to be disadvantages of this study.

\section{CONCLUSION}

The suspensory ligament incision (ligamentolysis) can be safely performed through the coronal ridge. This procedure also demonstrated improved results in terms of safety, operation time, retraction rate and cosmetic appearance, without any compromise in penile size gain, compared to the V-Y plasty technique. A strict patient selection criteria should be applied to avoid poor postoperative results. We are optimistic that the initial favorable outcomes of this circumcision phalloplasty procedure will be confirmed following evaluations in other centers and may become an established approach in the future.

\section{AUTHOR CONTRIBUTIONS}

$\mathrm{MN}$ was responsible for the concept and design of the study as well as the analysis of the data. He also gave the approval for the final version of the manuscript. KD was involved in the retrieval of the data and writing of the manuscript. BE was involved in the analysis of the data and performed intellectual modifications of the manuscript.

\section{COMPETING FINANCIAL INTEREST}

None of the authors declare any competing financial interest.

\section{ACKNOWLEDGMENTS}

We would like to thank Mrs Villy Rouchota (translator and interpreter) for her crucial contribution in identifying syntax and grammatical issues.

1 Austoni E, Guarneri A, Cazzaniga A. A new technique for augmentation phalloplasty: albugineal surgery with bilateral saphenous grafts. Eur Urol 2002; 42: 245-53.

2 Mondaini N, Ponchietti R, Gontero P, Muir GH, Natali A et al. Penile length is normal in most men seeking penile lengthening procedures. Int J Impot Res 2002; 14: 283-6.

3 Dillon BE, Chama NB, Honig SC. Penile size and penile enlargement surgery. Int J Impot Res 2008; 20: 519-29.

4 Ralph D, Gonzalez-Cadavid N, Mirone V, Perovic S, Sohn M et al. Trauma, gender reassignment, and penile augmentation. J Sex Med 2010; 7: 1657-67.

5 Vardi Y, Harshai Y, Gil T, Gruenwald I. A critical analysis of penile enhancement procedures for patients with normal penile size: surgical techniques, success, and complications. Eur Urol 2008; 54: 1042-50.

6 Levine L, Larsen S. Surgery for peyronie disease. Asian J Androl 2013; 15: 27-34.

7 Ponchietti R, Mondaini N, Bonafe M, Di Loro F, Biscioni S et al. Penile length and circumference: a study on 3,300 young Italian males. Eur Urol 2001; 39: 183-6.

8 Althof SE, Cappelleri JC, Shpilsky A, Stecher V, Diuguid C et al. Treatment responsiveness of the Self-Esteem and Relationship questionnaire in erectile dysfunction. Urology 2003; 61: 888-92.

9 Alter GJ. Reconstruction of deformities resulting from penile enlargement surgery. J Urol 1997; 158: 2153-7.

10 Wessells H, Lue TF, McAninch JW. Complications of penile lengthening and augmentation seen at 1 referral center. J Urol 1996; 155: 1617-20.

11 Roos H, Lissoos I. Penis lengthening. Int J Aesth Restor Surg 1994; 2: 89-96.

12 Li CY, Kayes O, Kell PD, Christopher N, Minhas S et al. Penile suspensory ligament division for penile augmentation: indications and results. Eur Urol 2006; 49: 72933.

13 Spyropoulos E, Christoforidis C, Borousas D, Mavrikos S, Bourounis M et al. Augmentation phalloplasty surgery for penile dysmorphophobia in young adults: considerations regarding patients selection, outcome evaluation and techniques applied. Eur Urol 2005; 48: 121-8.

14 Panfilov DE. Augmentative phalloplasty. Aesth Plast Surg 2006; 30: 183-97. 


\section{APPENDIX 1: SEAR QUESTIONNAIRE}

\section{Self-Esteem and Relationship (SEAR) questionnaire}

During the past 4 weeks

1. I felt relaxed about initiating sex with my partner

2. I felt confident that during sex, my erection would last long enough.

3. I was satisfied with my sexual performance.

4. I felt that sex could be spontaneous.

5. I was likely to initiate sex.

6. I felt confident about performing sexually.

7. I was satisfied with our sex life.

8. My partner was unhappy with the quality of our sexual relations.

9. I had good self-esteem.

10. I felt like a whole man.

11. I was inclined to feel that I am a failure.

12. I felt confident.

13. My partner was satisfied with our relationship in general.

14. I was satisfied with our relationship in general.

Response options:

$1=$ Almost never/never

$2=$ A few times (much less than half the time)

$3=$ Sometimes (about half the time)

$4=$ Most times (much more than half the time)

$5=$ Almost always/always

\section{APPENDIX 2}

A five-step questionnaire used preoperatively to estimate the personal relations, the self-esteem status, the quality of life and the expectations of surgical candidates

\begin{tabular}{|c|c|c|c|c|c|c|c|}
\hline & Question & Answer & & & & & \\
\hline 1 & $\begin{array}{l}\text { Does your perception of your penile size, even if it is } \\
\text { normal, affect your interactions with sexual } \\
\text { partners and professional associates? }\end{array}$ & Yes & $\square$ & No & $\square$ & I cannot say & $\square$ \\
\hline 2 & $\begin{array}{l}\text { Do you believe that male self-esteem and self-worth can } \\
\text { be affected by the 'adequacy' of a man's genitalia? }\end{array}$ & Yes & $\square$ & No & $\square$ & I cannot say & $\square$ \\
\hline 3 & $\begin{array}{l}\text { If penile enlargement surgery improves self-esteem } \\
\text { but other confidence issues are present, should these } \\
\text { issues be dealt with to change the quality of life? }\end{array}$ & Yes & $\square$ & No & $\square$ & I cannot say & $\square$ \\
\hline 4 & How much extra length do you expect? & $1-2 \mathrm{~cm}$ & $\square$ & $2-3 \mathrm{~cm}$ & $\square$ & more than $3 \mathrm{~cm}$ & $\square$ \\
\hline 5 & How much extra width do you expect? & $1-3 \mathrm{~cm}$ & $\square$ & $3-5 \mathrm{~cm}$ & $\square$ & more than $5 \mathrm{~cm}$ & $\square$ \\
\hline
\end{tabular}

